\title{
Visualization of aspirated flow pathlines in a thrombectomy catheter with three extra holes: A CFD study
}

\author{
Sajjad Soleimani \\ Laboratory of Biological Structure Mechanics, Department of Chemistry, Materials, and Chemical Engineering "Giulio Natta," Politecnico di \\ Milano, Milan, Italy
}

Email address:

zzsajad@yahoo.com

To cite this article:

Sajjad Soleimani. Visualization of Aspirated Flow Pathlines in a Thrombectomy Catheter with Three Extra Holes: A CFD Study. American Journal of Biomedical and Life Sciences. Special Issue: Numerical and Experimental Research in Cardiovascular Sciences.

Vol. 3, No. 2-2, 2015, pp. 1-5. doi: 10.11648/j.ajbls.s.2015030202.11

\begin{abstract}
The aspiration catheter design requires the good understanding of blood flow, based on a complex analysis of its hemodynamic characteristics. Computational fluid dynamics (CFD) is an important approach in studying the blood flow. In this paper the effect of extra upper holes - designed for the aspiration catheter - on the aspirated fluid pathlines are studied and discussed.
\end{abstract}

Keywords: Aspiration Catheter, Blood Flow, Computational Fluid Dynamics

\section{Introduction}

The analysis of blood hemodynamics implies the study of blood flow characteristics mostly based on clinical investigations, but in-vivo measurements are difficult and risk-posing, so simulation gains a lot of importance.

The numerical study of biomechanical characteristics of a realistic carotid artery using different inlet profiles leaded to the conclusion regarding a negative relation between velocity at several inner points of the internal carotid artery and velocity at the inlet of the common carotid artery [1]. The inlet boundary conditions were the data measured at the common carotid artery. The computational domain was generated using computed tomography.

A non-invasive method for performing in vivo measurements on blood velocity is 4D phase-contrast magnetic resonance angiography, on the basis of which a flow field with all its parameters can be simulated [2].

Other, performed study for predictive assessment of blood damage in bileaflet mechanical heart valves with pediatric sizing and flow conditions, using a scaled model of an adult-sized valve for the simulations [3].

New algorithms were validated for the efficient prediction of the hemodynamics in large arteries, relying on finite element simulation of the fluid-structure interaction between blood flow and arterial wall deformation of a healthy aorta [4].
Aiming to visualize the flow in the region of vertebral arteries junction in the origin of the basilar artery, simulations were also performed with the non-Newtonian model of blood [5].

In order to simulate blood flow and clot aspiration, the boundary conditions of the mathematical model should be carefully chosen. While vessel boundary can be set using various imaging techniques, such as computed tomography (CT), the segmentation process, which directly affects the accuracy, operates with algorithms and refinement methods. The isolation of interest vascular structure offers the inflow and outflow conditions. The flow results from 3D Navier-Stokes equations, more often for incompressible Newtonian fluid [6].

Simple coronary aspiration catheters are preferred to treat percutaneous coronary intervention (PCI) which could be followed by direct stenting if the vessel is occluded by both clot and plaque. The aspiration catheter access to a lesion in a coronary artery is through the femoral artery and then the catheter will be guided toward the occlusion inside the coronary artery. At this time the aspiration is applied via opening the valve connecting the pre-vacuumed syringe to the main lumen of the catheter. The aspiration will be terminated if the clot is already aspirated or five to seven attempts did not lead to any successful clot aspiration [7], 
meaning that, if the catheter is clogged by the clot, it should be removed and replaced by a new one, based on the interventionist's judgment, angiographic and clinical endpoints [8].

The paper describes the steps used in modeling the coronary aspiration catheter performance, explaining the method of acquisition of the coronary segment image and the numerical reliability of the model. Moreover the effect of each of extra holes on aspirated flow pathlines is studied.

\section{Modeling of the Coronary Aspiration Catheter Performance}

\subsection{Coronary Artery Imaging and Modeling}

To model the hemodynamic behavior of a clot during the aspiration process, a real topology and boundary conditions should be derived. Based on the tissue and blood properties X-ray, Magnetic Resonance and Ultra Sound are among the available imaging options.

However the disadvantages are the need for catheterization and radiation dose risks. Another method is the use of Computed Tomography (CT) scan by injecting contrast intravenously and rapidly capturing 3-D images with the help of multiple ring helical CT scanners. This method is less invasive but also leads to lower resolution images.

CT scan in combination with IntraVascular UltraSound (IVUS) can provide a 3-D image of lumen with appropriate resolution. The Magnetic Resonance Imaging technique (MRI) on the other hand does not require any ionizing radiation because it only uses magnetic spin relation properties of the tissues to distinguish them and, especially for imaging the blood vessel, the blood itself can be used as a contrast agent. This method is still with lower resolution comparing to X-ray method.

Creating a 3-D model out of 2-D images is a very typical approach. In this method a series of 2-D cross sectional images are recruited to reconstruct a 3-D representation mainly by positioning them following the real vessel centerline and then connecting the corresponding points around the circumference of the vessel through filtering techniques. If this is a segment of vessel without side branches, it is quite straightforward. If side branches are present, one can apply the same procedure for each side branch and then merge them through solid modeling operation $[9,10]$.

Although CT scans are commonly used in the clinical practice to image vessels, this method cannot create a 3-D image of coronary vessel suitable to be directly imported in Computational Fluid Dynamics (CFD) packages. The 2-D images extracted through intravascular ultrasound (IVUS) are combined with coronary computed tomography angiography (CTA) images and eventually a 3-D model emerges. The procedure passes through several manipulating and interpolation. The extracted image, converted to a "stl" file and merged with catheter design models in order to be meshed (geometrically discretized) with ANSYS ICEM CFD 12.1 (ANSYS, Inc., Caconsburg, PA, USA), the same commercial software employed for numerical analysis. The adopted 3D model of the coronary bifurcation resulted from a mixed method.

To model the viscosity of blood, the non-newtonian Bird-Carreau model is used:

$$
\mu=\mu_{\infty}+\left(\mu_{0}-\mu_{\infty}\right)\left(1+\lambda^{2} \dot{\gamma}^{2}\right)^{\frac{n-1}{2}}
$$

where $\mu_{0}=0.56 \mathrm{~Pa}, \mu_{\infty}=0.0345 \mathrm{~Pa}, \lambda=3.313 \mathrm{~s}$, and $\mathrm{n}=0.3568$ $[11,12]$.

\subsection{Numerical Reliability}

In many cases there are no alternative ways to predict the results and to understand their details by means of experimental or analytical studies. This becomes more critical when one must deal with small-scale devices. Furthermore, once a reliable numerical model for a specific problem has been created it will be easily - and much cheaply - applicable to similar cases, especially when performance optimization is the target. This is mainly due to the huge number of parameters which may govern the problem. Thus in many complex problems, the numerical approach leads to less expensive and more detailed solutions and offers better chances of controlling the parameters in order to optimize the performance.

All the above advantages may turn into disadvantages if an appropriate approach is not chosen.

The grid resolution, time step size and the residuals reduction are examined to predict the reliability of the numerical study.

\subsubsection{Grid Independency Study}

The grid independency study was performed by comparing meshes with multiplied denser grids with each, because the final goal is to achieve the results regardless of the mesh density.

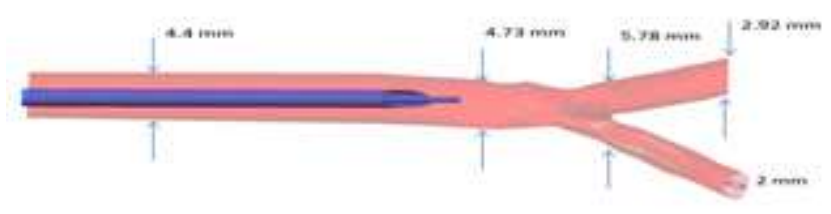

Figure 1. Scheme of the catheter in a coronary bifurcation, The adopted coronary model is the courtesy of the European Bifurcation Club, Lisbon, 2011 .

Two meshes with total grid amount of 1.011 .000 (simulation mesh) and 7.209.542 (control mesh) cells were considered and compared with each other. The coarser mesh is however denser in the area near the catheter entrance to capture the fluid instantaneous curvature. Cross sections of those meshes are illustrated in Fig.2. The pressure difference of $20 \mathrm{KPa}$ between the catheter outlet and the vessel outlets cross section is applied to drive the clot movement. As the main aim of this study is to model and evaluate the aspiration ability of each catheter, the remained volume of clot is considered as a critical parameter to be 
studied for the grid and time step study.

The comparison of the error on the two mesh sizes of simulation mesh and control mesh over time indicated an average error of $2.3 \%$ for the 20 seconds of simulation, showing the results accuracy.

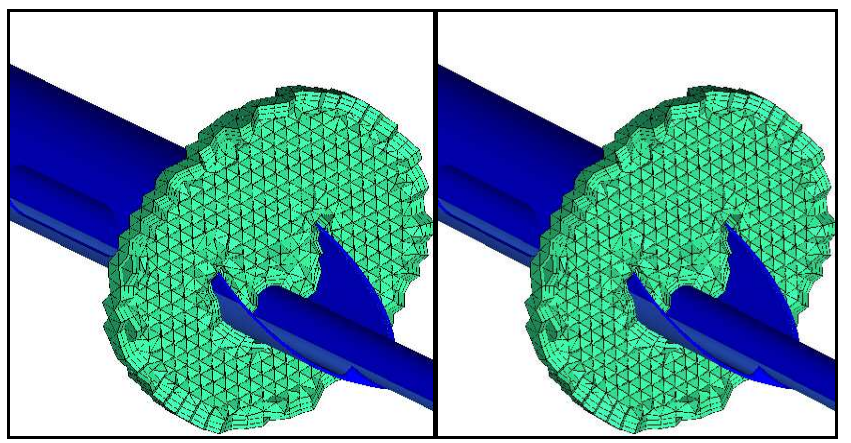

(a)

(b)

Figure 2. 3D cross section of the simulation mesh (a) and control mesh (b).

\subsubsection{Time Step Independency Study}

In order to capture the transient simulation of clot aspiration, a time marching simulation is carried out. For the understanding of the chosen time step, two time steps, different in one order of magnitude were considered and compared with each other: $\Delta \mathrm{t}=0.01$ and $0.001 \mathrm{~s}$.

It is seen that by 10 times reducing the time step, the average error is $1.13 \%$ for the first $14 \mathrm{~s}$. Thus the time step of $0.01 \mathrm{~s}$ is appropriate for the modelling.

Table 1. Remained volume of clot, $\mathrm{mm}^{3}$ for $\Delta t=0.01$ and $0.001 \mathrm{~s}$

\begin{tabular}{llll}
\hline Time, $\mathbf{s}$ & $\boldsymbol{\Delta t}=\mathbf{0 . 0 1} \mathbf{s}$ & $\boldsymbol{\Delta} \mathbf{t}=\mathbf{0 . 0 0 1} \mathbf{~ s}$ & Error, $\%$ \\
\hline 2 & 47.5 & 46.1 & 2.9 \\
4 & 44.5 & 43.63 & 2.3 \\
6 & 42.4 & 42.7 & 0.6 \\
8 & 41.5 & 41.6 & 0.4 \\
10 & 40.7 & 41 & 0.7 \\
12 & 40.2 & 40.4 & 0.7 \\
14 & 39.5 & 39.6 & 0.3 \\
\hline
\end{tabular}

\subsubsection{Appropriate Reduction of Residuals}

The scaled residual is defined as follows

$$
\frac{\sum_{i=1}^{M}\left|a_{p} \psi_{p}-\sum_{j=1}^{n_{i}} a_{j} \psi_{p_{j}}-s\right|}{\sum_{i=1}^{M}\left|a_{p} \psi_{p}\right|}
$$

The residual should be reduced to minimum, so the residual values of velocity and volume of fluid phase dropped below $10^{-4}$ were considered appropriate.

\subsection{Boundary Conditions}

In order to model a still initial condition due to the blood blockage caused by clot in the coronary vessel, the same value of pressure are considered at the vessel main and daughter branches outlet cross section. The vacuum is applied through the pressure difference between the catheter outlet and vessel branches outlet cross sections. The pressure of $10 \mathrm{kPa}$ and -10 $\mathrm{kPa}$ are applied on vessel branches and catheter tip boundary condition, respectively.

\section{Results}

In the simulation, fluid (blood and clot) pathlines are visible and thus easily investigated.

It should be noted that due to the length of the catheter lumen, only a fraction of pressure provided by the syringe is introduced to the clot location [13]. Thus although a high pressure is provided by the syringe only a $-10 \mathrm{kPa}$ pressure is introduced at the catheter outlet which creates a $20 \mathrm{kPa}$ pressure difference to aspirate the clot.

The fluid path line for the aspiration of clot is shown in Fig. 3. With the simulation visualization, it became obvious which catheter hole is more efficient and which the hole position is useless, as well as the aspiration efficiency for the clot. Also, synergic effects of catheter design aspects could be studied.

The pathlines visualization is depicted for the aspiration of clot with low and medium viscosity value. The effect of the upper holes is accordingly studied over time. Originally the upper holes are designed to enhance the aspiration of the part of clot which may not be aspirated through the main front hole, however it is very important to study whether such holes are able to appropriately aspirate clot.

In the Figure the sequences of the pathlines of aspiration of clot over time are presented. At the beginning the main front hole and the distal hole have more effect on the aspiration (the main front hole is the hole designed at the head of the tip of the catheter and the distal hole is the one closest to the main front hole). Over time by movement of clot toward the proximal parts of the catheter, the distal holes as well as the main front hole have no role any more on the aspiration; instead the proximal holes take role of the aspiration. However it should be considered that if there is not any upper hole, the clot may not travel to the proximal parts of the catheter, in contrast, by the absence of extra hole (the upper holes) the catheter may not be able to prevent clot lodging around its tip.

\section{Discussions}

\subsection{Main Findings}

The streamlines depict the role of each hole and specify their effect on clot aspiration over time for aspirating low and medium viscosity clot. It indicates that, as long as during the aspiration, part of clot may not be aspirated by the main front hole, the upper hole may facilitate the clot aspiration. The upper holes contribute in clot aspiration one after the other one from distal part to the proximal part, when the clot mass moves toward the proximal part over time.

However it should be noted that this arrangement (all holes 
in a line on the upper side of the catheter similar to Diver CE KIT catheter) is not the best arrangement. Moreover, the use of extra hole is preferable only for low and medium viscosity clot and not for the aspiration of clot with high viscosity [14].

\subsection{Limitations}

In order to improve the model, a vast variety of clot mechanical properties (including clot with various viscosities) should be studied. Furthermore various boundary conditions should be considered. The upper hole should be also studied for varies sizes in order to consider the optimum case. So that a more generalized conclusion could be derived accordingly.



Time $1 \mathrm{~s}$

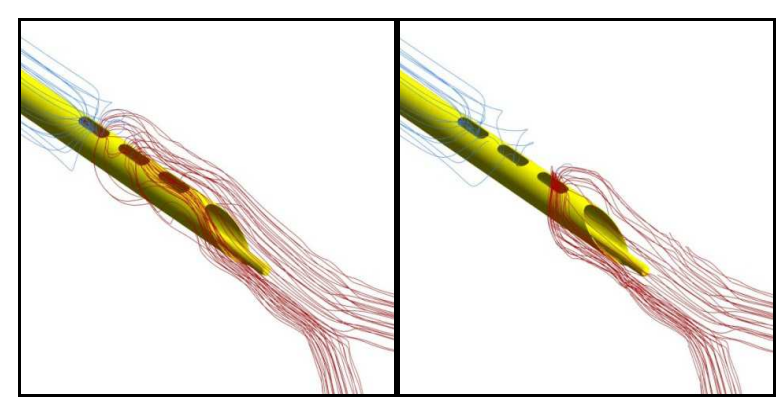

Time $3 \mathrm{~s}$

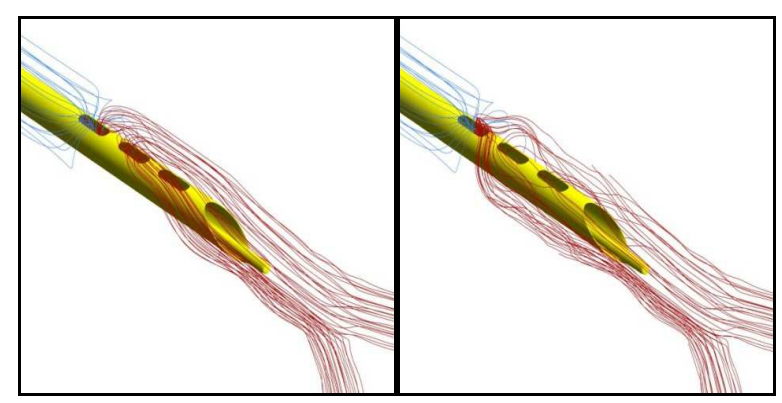

Time $5 \mathrm{~s}$



Time $7 \mathrm{~s}$

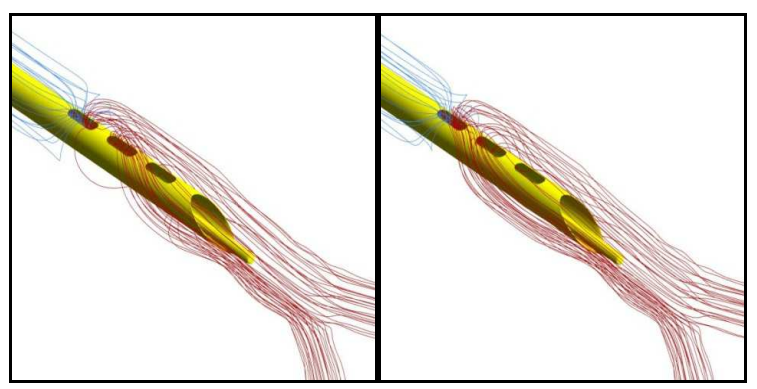

Time $10 \mathrm{~s}$

Figure 3. Pathlines of the aspirated blood and clot over time, left column: low viscosity clot, right column: medium viscosity column.

\section{Conclusions}

Flow visualization in the case of aspiration catheter performed with ANSYS had reasonable values for error and residuals. While residuals of velocity and fluid phase volume were below $10^{-4}$, errors for simulation and control mesh had a $2.3 \%$ mean value.

The method developed for inspecting flow data on aspiration catheter designs is meaningful due to the characteristics of the data and the level of errors. The integral curves used in order to represent pathlines were correctly chosen to imagine trajectories in blood flow. Therefore, the risk of accumulated errors at each integration step of the pathlines was minimized.

The aspiration catheter design requires the good understanding of blood flow, based on a complex analysis of its hemodynamic characteristics. Computational fluid dynamics is an important approach in studying the blood flow. The paper describes the steps used in modeling the coronary aspiration catheter performance, explaining the method of acquisition of the coronary segment image and the numerical reliability of the model achieved with minimum errors and residuals. The effect of each of three extra upper holes on the fluid pathlines is also studied.

\section{Acknowledgements}

This research is funded by the European Commission through MeDDiCA ITN (grant agreement PITN-GA-2009-238113).

\section{References}

[1] S. Piskin, M. S. Celebi, "Analysis of the effects of different pulsatile inlet profiles on the hemodynamical properties of blood flow in patient specific carotid artery with stenosis", Computers in Biology and Medicine, vol.43, pp.717-728, 2013

[2] D. I. Zuleger, D. Poulikakos, A. Valavanis, S. S. Kolliadis, "Combining magnetic resonance measurements with numerical simulations - Extracting blood flow physiology information relevant to the investigation of intracranial aneurysms in the circle of Willis", Int. J. of Heat and Fluid Flow, vol. 31, pp.1032-1039, 2010. 
[3] B. M. Yun, D. B. EcElhinney, S. Arjunon, L. Mirabella, C. K. Aidun, A. P. Yoganathan, "Computational simulations of flow dynamics and blood damage through a bileaflet mechanical valve scaled to pediatric size and flow", J. Biomech., vol. 47, pp.3169-3177, 2014.

[4] P. Crosetto, P. Reymond, S. Departis, D. Kontaxakis, N. Stergiopulos, A. Quarteroni, "Fluid-structure interaction simulation of aortic blood flow", Computers \& Fluids, vol. 42, pp.46-57, 2011.

[5] K. Jozwik, D. Obidowski, "Numerical simulations of the blood flow through vertebral arteries" J. Biomech., vol. 43, pp.177-185, 2010.

[6] B. Preim, C. P. Botha, "Visual Computing for Medicine", 2nd ed., 2014, pp. 665-713.

[7] Z. Han-jun, Y. Hong-bing, W. Jian, S. Li, Qing-xiang, L. L. Shi-ying, C. Yun-peng, W. Zheng, Z. Xiao-jiang, Z. Yong, Z. Bin, "Comparison of Diver CE and ZEEK manual aspiration catheters for thrombectomy in ST-segment elevation myocardial infarction", Chinese Medical Journal, 2009, 122(6): 648-654.

[8] F. Burzotta, C. Trani, E. Romagnoli, F. Belloni, G. Biondi-Zoccai, M.A. Mazzari, M. Vita, F. Giannico, B. Garramone, G. Niccoli, A.G. Rebuzzi, R. Mongiardo, G. Schiavoni, F. Crea, A Pilot Study With a New, Rapid-Exchange, "Thrombus-Aspirating Device in Patients With Thrombus-Containing Lesions: The Diver C.E. Study", Catheterization and Cardiovascular Interventions, 2006, 67: 887-893.
[9] D.A. Steinman, "Image-Based Computational Fluid Dynamics Modeling in Realistic Arterial Geometries", Annals of Biomedical Engineering, 2002, 30: 483-497.

[10] A.G. Giessen, M. Schaap, F.J.H. Gijsen, H.C. Groen, T. Walsum, N.R. Mollet, J. Dijkstra, F.N. Vosse., W.J. Niessen, P.J. Feyter, A.F.W. Steen, J.J. Wentzel, "3D fusion of intravascular ultrasound and coronary computed tomography for in-vivo wall shear stress analysis: a feasibility study, International Journal of Cardiovascular Imaging", 2010, 26: 781-796.

[11] Y.I. Cho, K.R. Kensey, "Effects of the non-Newtonian viscosity of blood on flows in a diseased arterial vessel. Part 1: steady flows", Biorheology 1991;28:241-62.

[12] B.M. Johnston, P.R. Johnston, S. Corney, D. Kilpatrick, "Non-Newtonian blood flow in human right coronary arteries: steady state simulations", J Biomech 2004;37:709-20.

[13] G. Romero, M.L. Martinez, J. Felez, G. Pearce, N.D. Perkinson, "Analysis and design of a thrombectomy device by using simulation techniques", GProceedings of the IMProVe 2011 International conference on Innovative Methods in Product Design June 15 th -17 th, 2011, Venice, Italy.

[14] S. Soleimani, G. Dubini, G. Pennati, "Possible Benefits of Catheters With Lateral Holes in Coronary Thrombus Aspiration: A Computational Study for Different Clot Viscosities and Vacuum Pressures", Artificial Organs 2014, doi: 10.1111/aor.12274 